\title{
Single Atom Detection Through HAADF-STEM and EELS/EDX Characterization of Fluorophore Ru(bpy)32+ for Optical DNA-Chip Applications
}

\author{
G. Nicotra ${ }^{1}$, E.L. Sciuto ${ }^{1}$, M.F. Santangelo ${ }^{2}$, G. Villaggio ${ }^{3}$, F. Sinatra ${ }^{4}$, C. Bongiorno ${ }^{1}$, S. Libertino ${ }^{1}$ \\ ${ }^{1}$ CNR-IMM, Strada VIII, 5, 95121 Catania, Italy. \\ 2 Distretto Tecnologico Sicilia Micro e Nano Sistemi, Catania, Italy \\ ${ }^{3}$ Dipartimento G. F. Ingrassia, Università degli Studi di Catania, Catania, Italy \\ ${ }^{4}$ Dipartimento di Scienze Biomediche, Università degli Studi di Catania, Catania, Italy
}

Optical DNA-chip is widely used to study genome, gene expression and genetic diseases [1] We can divide it into three basic components: the sensing element or probe specific for target gene (single strand DNA); the labeling system, provided by conventional fluorophore CY5 (indodicarbocyanine) [2]; the optical detector, i.e. imagers or scanners. The labeling organic fluorophores exhibiting absorption/emission peaks very close, $\sim 20 \mathrm{~nm}$ apart. Unfortunately, their prolonged exposure to laser light causes photo bleaching [3]. Moreover, their short lifetime, below 3 ns [4], could be an obstacle for the design of a miniaturized device, since a complex and fast (in the $\mathrm{GHz}$ range) electronic management system may be needed. In order to achieve the goal of a miniaturized, cheap and "simple" optical biosensor, we characterized an organometallic fluorophore, the tris(2,2'bipyridyl)ruthenium(II) (Ru(bpy)32+). Our studies revealed that Ru(bpy)32+ emission properties (peak and lifetime) strongly depend from the chemical and physical environment. In particular we found an additional emission peak at $590 \mathrm{~nm}$ which dominates in the dried form regardless of the surface used to deposit the sample, while the lifetime value increases of a factor ten to 30 ns. In order to understand the relation between the fluorophore form and its optical properties we firstly performed a full structural characterization through Low-Energy Scanning Transmission Electron Microscopy (STEM), operated at $60 \mathrm{keV}$, by using a Cs corrected TEM JEOL ARM 200F. STEM analysis, of pure drop casted water solution containing Ru(bpy)32+ Thanks the very high sensitiveness of HAADF no negative staining, such as heavy uranyl acetate, was necessary. STEM observation in conjunction with chemical mapping performed by Electron Energy Loss Spectroscopy (EELS) and Energy Dispersive X-Ray Analysis (EDX), allowed us to identify fluorophore clusters of few nanometers, Figure 1, and to find a possible explanation for optical properties of the dried form. We than moved at the $200 \mathrm{keV}$ of beam energy, and for the first time we observed the single atoms of $\mathrm{Ru}$, which are the direct evidence of the actual presence and the amount of molecules and their distribution on the substrate, Figure 2,. Motion of such single atoms has been also observed and studied under the electron beam radiance. For this study, a state-of-the-art aberration-corrected microscope installation at Beyond-Nano sub-Ångstom Lab, in Catania, Sicily, Italy, has been used. This consists of a probe corrected STEM microscope equipped with a C-FEG and a fully loaded GIF Quantum ER as EELS spectrometer. This particular installation is capable to deliver a probe size of $0.68 \AA$ at $200 \mathrm{kV}$, and $1.1 \AA$ at $60 \mathrm{kV}$. Chemical mapping were extracted from the simultaneous acquired EELS and EDX spectra, by using the Spectrum Imaging mode. 


\section{References}

[1] R. W. Ye, T. Wang, L. Bedzyk and K. M. Croker, “Applications of DNA microarrays in microbial systems”, J. Microbiol. Methods 47-257, 2001..

[2] M. Schena, D. Shalon, R. W. Davis and P. O. Brown, "Quantitative monitoring of gene expression patterns with a complementary DNA microarray”, Science 270, 467-470, 1995.

[3] J. R. Lakowicz, "Radiative Decay Engineering: Biophysical and BiomedicalApplications”, REVIEW Analytical Biochemistry Vol. 298, pp. 1-24, 2001.

[4] M. F. Santangelo, R. Pagano, S. Lombardo, E. L. Sciuto, D. Sanfilippo, G. Fallica, F. Sinatra, A. C. Busacca, S. Libertino, "SiPM as novel Optical Biosensor: transduction and applications”, submitted for publication IEEEXplore, 2014.

[5] This work was performed at Beyondnano CNR-IMM, which is supported by the Italian Ministry of Education and Research (MIUR) under project Beyond-Nano (PON a3_00363).

Figure 1. a) Z-Contrast HAADF STEM image with no negative contrast. b) EELS map of N K1 edge; c) EDX chemical map of Ru L edge; Chemical maps shows that Nitrogen and Ruthenium are localized on the region occupied by the bright contrast. d) HAADF showing the presence fluorophore clusters of few nanometers
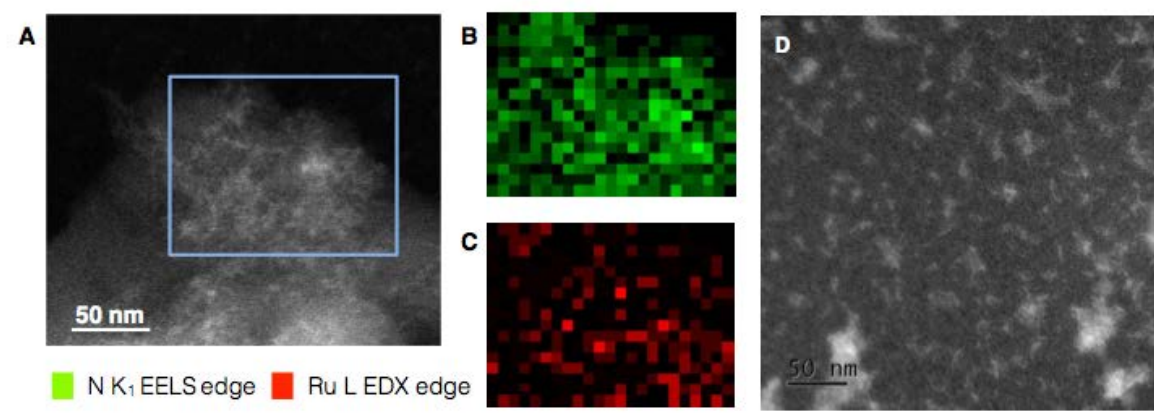

Figure 2. a) Plain carbon supporting film gold grid and corresponding EDX spectrum b); c) carbon supporting film gold grid containing the fluorophore $\mathrm{Ru}(\mathrm{bpy}) 32+$, bright spot are single $\mathrm{Ru}$ atoms, and corresponding EDX spectrum d)
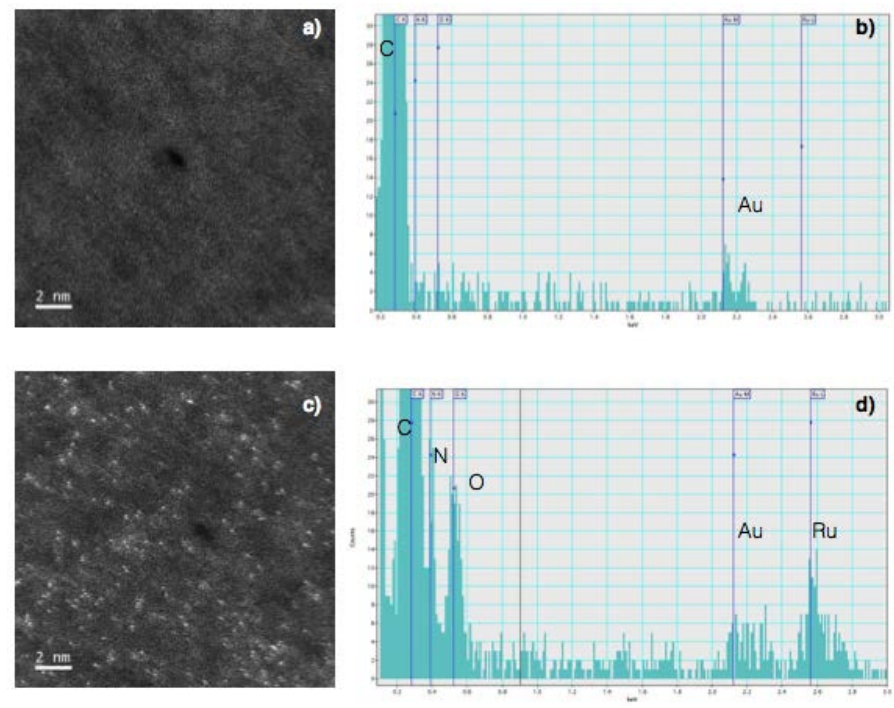